\title{
Survey of current practices among US epileptologists of antiepileptic drug withdrawal after epilepsy surgery
}

\author{
Christa B. Swisher, Saurabh R. Sinha* \\ Department of Medicine (Neurology), Duke University Medical Center, USA
}

\section{A R T I C L E I N F O}

\section{Article history:}

Received 24 September 2012

Revised 27 November 2012

Accepted 27 November 2012

Available online 8 January 2013

\section{Keywords:}

Epilepsy

Antiepileptic drugs

Epilepsy surgery

Mesial temporal sclerosis

\begin{abstract}
A B S T R A C T
In order to identify the current practices of antiepileptic drug (AED) withdrawal after epilepsy surgery, a survey was administered to 204 adult and pediatric epileptologists. The responses from 58 epileptologists revealed wide variations regarding the time course and extent of AED withdrawal after successful epilepsy surgery. For most of the epileptologists, the likelihood of the surgery being successful is an important factor in determining whether or not AEDs are tapered. Most of the respondents started to taper AEDs more rapidly than suggested by previous reports. The majority of the epileptologists were able to stop all AEDs completely in a substantial number of patients. The most important factors considered when deciding to taper AEDs were the presence of ongoing auras and the occurrence of postoperative seizures prior to seizure remission. In the absence of data from well-designed prospective trials, such survey results can inform practice and, hopefully, aid in the design of future trials.
\end{abstract}

Published by Elsevier Inc.

\section{Introduction}

Temporal lobectomy and other forms of resectional epilepsy surgery are a treatment option for patients with focal epilepsy refractory to medical treatment. The median seizure-freedom rate from temporal lobectomy for mesial temporal sclerosis is 70\% [1] and is somewhat lower for extra-temporal resections and for patients with non-lesional epilepsy [2]. Although the primary goal of epilepsy surgery is seizure control, reduction or discontinuation of antiepileptic drugs (AEDs) is also a significant goal of patients and epileptologists after epilepsy surgery. Patients remaining on AEDs after successful resectional epilepsy surgery may continue to experience adverse side effects from these medications. Common side effects from AEDs include dizziness, sedation, fatigue, cognitive problems and cost. These side effects are particularly common when AED polytherapy is used, which is common in patients undergoing epilepsy surgery. Pooled data from surgical case series indicate that a significant portion of patients achieve seizure freedom as well as discontinuation of all AEDs [3]. Overall, 22\% of 936 patients in 9 studies who underwent epilepsy surgery achieved seizure freedom and AED freedom with a mean follow-up of 6.9 years; the rate was slightly higher for children (27\%) compared with adults (19\%).

Due to these reasons, for many patients and some physicians, the ultimate goal of epilepsy surgery is total seizure freedom and withdrawal of all AEDs, though no guidelines exist regarding AED withdrawal after epilepsy surgery. The published data on this topic

\footnotetext{
* Corresponding author at: 299B Hanes House, 330 Trent Drive, Box 102350, Duke University Medical Center, Durham, NC 27710, USA. Fax: + 19196848955.

E-mail address: saurabh.sinha@duke.edu (S.R. Sinha).
}

are limited, consisting mainly of case series, some fairly large. In prospective studies, AED tapering has usually not been randomized; instead all eligible patients are offered the chance to taper AEDs, and of that subset who undergo tapering of AEDs, characteristics of the group having seizure recurrence is compared to those that do not. Studies have shown that AED withdrawal after epilepsy surgery is more likely to be successful if the patient has been seizure-free with AEDs for a minimum of one year after surgery [4]. However, there are other factors that may be important when deciding if and when to withdraw AEDs, including normal versus abnormal preoperative MRI, duration of epilepsy, and epileptiform findings on postoperative EEGs [5,6].

There is a lack of controlled data regarding optimal management of AEDs after epilepsy surgery [7]. Even at our Epilepsy Center, where 3 adult and 2 pediatric epileptologists collaborate closely in the evaluation and management of patients undergoing surgery for intractable seizures, we noticed a wide variety of practices in tapering of AEDs after successful epilepsy surgery. In order to identify the current practices of AED withdrawal after epilepsy surgery, we surveyed adult and pediatric epileptologists. In the absence of data from well-designed prospective trials, such survey results can inform practice; furthermore, they will, hopefully, aid in the design of future trials.

\section{Methods}

This study was approved by the Duke Institutional Review Board. To identify current practices regarding AED withdrawal after epilepsy surgery, an electronic request to participate in the survey was sent to 204 epileptologists identified through membership in the National 
Table 1

Survey questions.

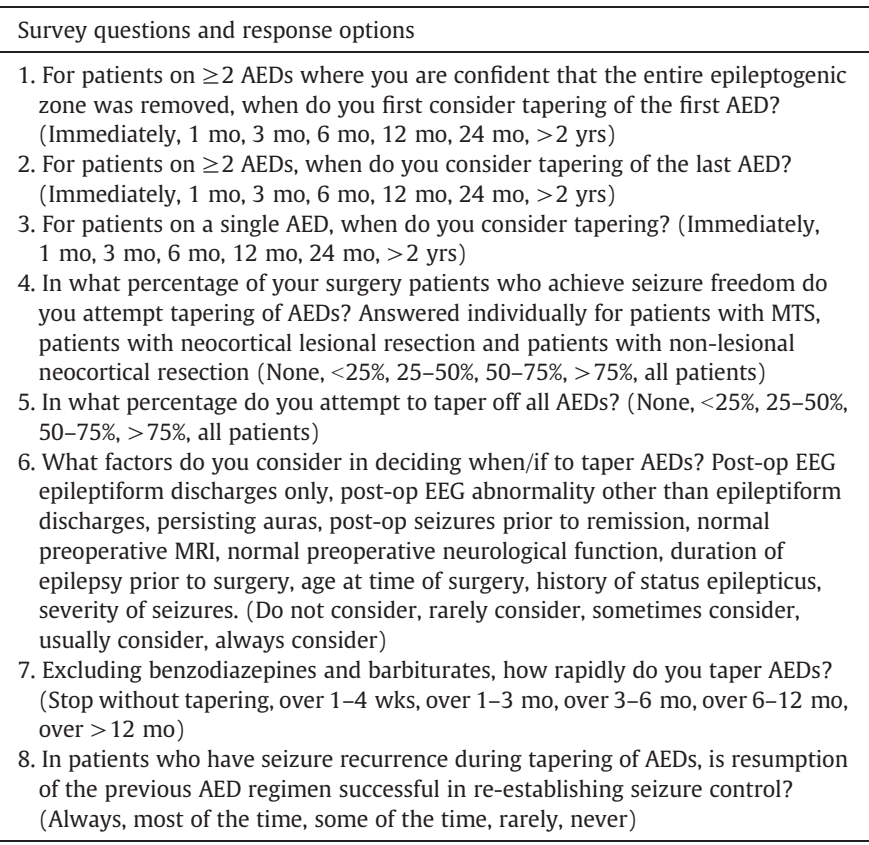

Association of Epilepsy Centers. The members who chose to participate completed a 10-15-minute online survey on Survey Monkey (www.surveymonkey.com). The survey was completed anonymously. The survey contained 11 multiple choice questions evaluating the type and duration of their epilepsy practice, the timing of AED withdrawal, the speed of AED withdrawal and the factors that come into consideration when deciding if AED withdrawal should be undertaken (Table 1).

\section{Results}

The survey was completed by 58 epileptologists (response rate of $28 \%$ ). Adult epileptologists comprised $66 \%$ of the respondents, with $14 \%$ being pediatric and $21 \%$ caring for both adult and pediatric patients. The majority ( $85 \%$ ) had $>10$ years of experience in treating patients with epilepsy with $79 \%$ handling more than 10 epilepsy surgery cases per year. All 58 epileptologists responded to each question, with exception of one respondent who did not answer question \#4 and also one respondent who did not answer part of question \#6 (all of the factors in question \#6 were answered except for severity of seizures).

The majority of the survey respondents attempted AED tapering in $50-100 \%$ of their patients who underwent successful epilepsy surgery and were seizure-free. In addition, the epileptologists were more likely to attempt AED withdrawal in patients who underwent lesional neocortical resection and anterior temporal lobectomy for mesial temporal sclerosis rather than in patients who underwent non-lesional neocortical resection (see Fig. 1).

Table 2 shows preferences for the timing of AED withdrawal when the entire epileptogenic zone was believed to have been removed for patients receiving AED polytherapy and monotherapy. For patients on $\geq 2$ AEDs who underwent successful epilepsy surgery, the majority of the epileptologists preferred to initiate AED withdrawal after 6 months (39\%) or after 12 months (28\% of the respondents). However, a significant number $(10.5 \%)$ started tapering immediately. This is in contrast to patients on AED monotherapy where the epileptologists preferred to wait longer to initiate tapering (12 months, $28 \%$; 2 years, $31 \%$; $>2$ years, $34.5 \%$ ). Most of the survey participants preferred to wait until 2 years or greater to initiate tapering of the last AED for patients receiving polytherapy at the time of their surgery. To assess how often AEDs are completely discontinued, we asked the epileptologists how often they taper off all AEDs after epilepsy surgery. The most common response (35\%) was that complete tapering off of all AEDs was completed in $<25 \%$ of their patients. An additional $22 \%$ of the epileptologists responded that they attempt complete AED discontinuation in 25-50\% of their patients, with $20 \%$ of the epileptologists attempting this in $50-75 \%$ of their patients.

The main factors that impacted decisions regarding AED withdrawal after epilepsy surgery appear to be persistent auras and if post-operative seizures occurred prior to seizure freedom with $83 \%$ usually and $69 \%$ of epileptologists always or usually considering these factors. These were followed by history of status epilepticus, severity of seizures, and presence of epileptiform discharges on the postoperative EEG, with approximately $50 \%$ of the epileptologists usually or always considering these factors. Patient age at the time of surgery, duration of epilepsy, presence of a normal preoperative MRI, presence of normal preoperative neurological functioning, and presence of an abnormal postoperative EEG other than epileptiform discharges were factors that were of lesser importance to the neurologists when deciding to taper AEDs (Fig. 2).

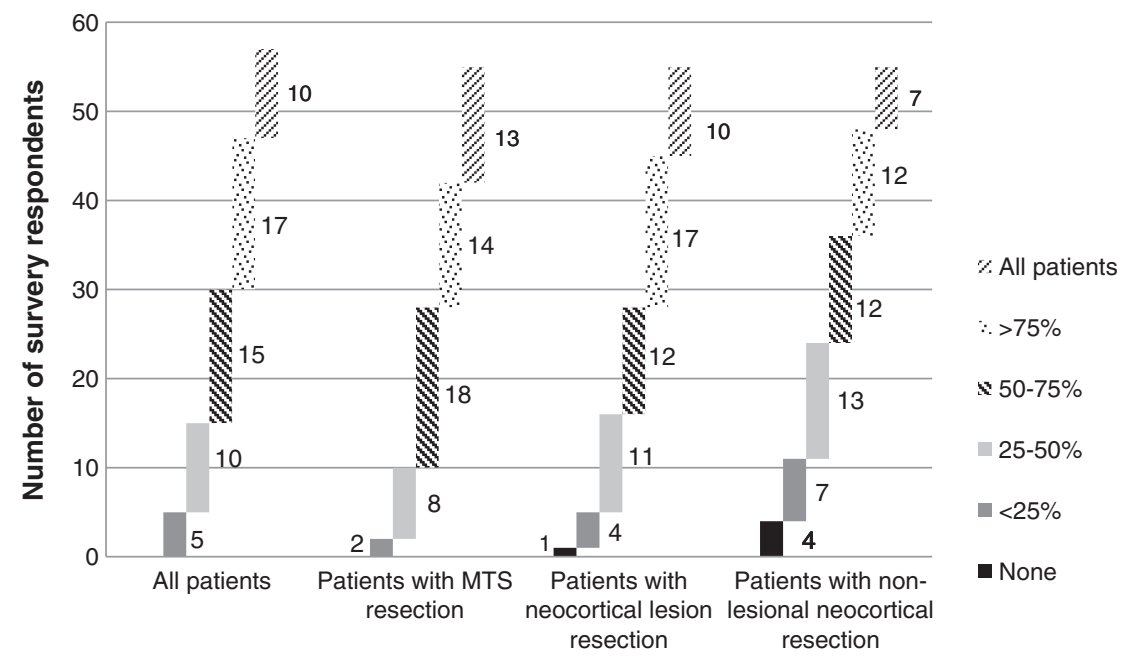

Fig. 1. For patients with different types of resection, percentage of patients in whom tapering of any AED was attempted. 
Table 2

Timing of AED withdrawal after successful epilepsy surgery for patients on $\geq 2$ AEDs and for patients on AED monotherapy.

\begin{tabular}{lccc}
\hline & $\begin{array}{l}\text { Initiation of AED } \\
\text { taper for patients } \\
\text { on } \geq 2 \text { AEDs }\end{array}$ & $\begin{array}{l}\text { Taper of last AED } \\
\text { for patients } \\
\text { on } \geq 2 \text { AEDs }\end{array}$ & $\begin{array}{l}\text { Taper of AED } \\
\text { for patients on } \\
\text { monotherapy }\end{array}$ \\
\hline $\begin{array}{l}\text { Immediately } \\
1 \text { month }\end{array}$ & $6(10.5 \%)$ & $1(1.7 \%)$ & $0(0 \%)$ \\
3 months & $3(0 \%)$ & $0(0 \%)$ & $0(0 \%)$ \\
6 months & $22(38.6 \%)$ & $0(0 \%)$ & $0(0 \%)$ \\
12 months & $16(28.1)$ & $1(1.7 \%)$ & $4(6.9 \%)$ \\
2 years & $6(10.5 \%)$ & $7(12.1 \%)$ & $16(27.6 \%)$ \\
$>2$ years & $4(7.0 \%)$ & $21(36.2 \%)$ & $18(31.0 \%)$ \\
\hline
\end{tabular}

We also asked about how rapidly an AED was tapered. The majority (55\%) of the survey respondents tapered AEDs over the course of 1-3 months. This was followed by a taper over 3-6 months (22\%), over 6-12 months (14\%), over $>12$ months (7\%), and over 1-2 weeks (2\%). No epileptologists stopped AEDs without a taper.

For patients who have seizure recurrence during the taper of AEDs, $76 \%$ of the epileptologists felt that resumption of their previous AED regimen controlled the seizures most of the time. Twenty-one percent felt resumption of their previous AED regimen controlled the seizures some of the time. An additional 2\% felt this always controlled the seizures, and another $2 \%$ felt this never controlled the patient's seizures after the occurrence of the breakthrough seizures during AED tapering.

\section{Discussion}

This survey of 58 adult and pediatric epileptologists reveals wide variations among epileptologists regarding the time course and extent of AED withdrawal after successful epilepsy surgery. Despite the differences in the timing and speed of AED withdrawal, all of the survey respondents stated that they do attempt AED tapering in some of their patients. The majority (74\%) of the respondents attempt an AED taper in at least half of their patients who are seizure-free after epilepsy surgery. A minority (17.5\%) attempt an AED taper in all of their patients. The respondents are more likely to attempt an AED taper in patients who underwent surgery for mesial temporal sclerosis rather than in patients with neocortical lesion resection. However, one retrospective study found that AED withdrawal was attempted in $88.7 \%$ of patients after undergoing extratemporal epilepsy surgery [8], suggesting that AED withdrawal rates may be just as common with patients who underwent extratemporal epilepsy surgery. The epileptologists are least likely to attempt an AED taper for patients who underwent non-lesional neocortical resection. This suggests that for most of the epileptologists, the likelihood of the surgery being successful is an important factor in determining whether or not AEDs are tapered - those patients with characteristics that suggest a high likelihood of complete seizure control after surgery are most likely to have their AEDs tapered.

These results are in general agreement with a previous survey of US epileptologists [9] and a recent survey of Canadian epileptologists [10]. In the former, $62 \%$ of the respondents said they waited $>2$ years of seizure freedom prior to tapering the last AED; $9 \%$ reported never tapering the last AED. This study did not address when tapering of patients on multiple AEDs was initiated or how rapidly drugs were tapered. In the Canadian survey, for patients on multiple AEDs, $21 \%$ of the respondents started to reduce AEDs before 6 months and an additional 50\% did so between 6 and 11 months [10]. Most of the respondents waited at least 2 years before tapering the last AED, although 21\% reported doing so between 6 and 11 months.

In our survey, most of the respondents started to taper AEDs more rapidly than suggested by previous reports [3], which recommended waiting for 1 year. On the other hand, a study by Schiller et al. supports the practice of an early AED taper and found that seizure recurrence after AED withdrawal was unrelated to the duration of the seizure-free period after surgery [6]. In the European TimeToStop observational study that included 766 children, the median time for initiation of AED taper was 12.5 months, suggesting that American and Canadian epileptologists begin AED tapering earlier than their European colleagues [11]. More importantly, the TimeToStop study found that early AED withdrawal did not affect long-term seizure outcome [11]. In our survey, a significant number of the respondents (45.6\%) preferred to wait even longer (12 months or more) to initiate AED tapering in patients on polytherapy. Conversely, for patients on monotherapy, most of the epileptologists preferred to wait significantly longer before initiating an AED taper, with approximately equal percentages preferring to initiate the taper at 12 months, at 2 years and at $>2$ years. The majority of the epileptologists were able to stop all AEDs completely in a substantial number of patients.

After the type of surgery and underlying abnormality, the most important factors when deciding to taper AEDs were the presence of ongoing auras and post-operative seizures prior to seizure remission, respectively, with epileptologists always or usually taking these factors into consideration. However, previous studies have not consistently shown persistent auras and rare postoperative seizures to have an impact on recurrence rate $[12,13]$. Studies evaluating the risk factors for seizure recurrence after successful epilepsy surgery are conflicting and

\section{What factors do you consider in deciding when/if to taper AEDs in patients after epilepsy surgery?}

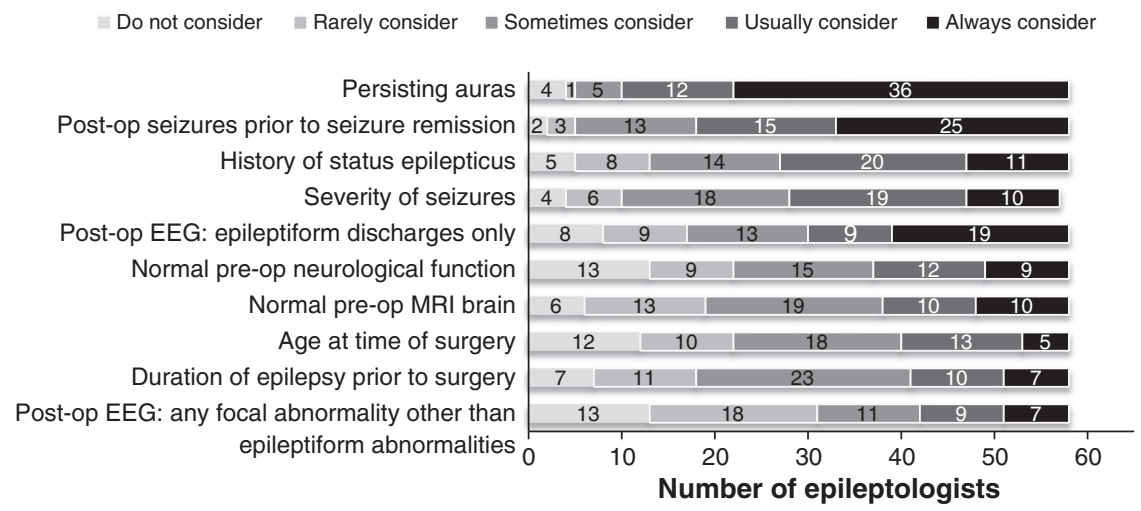

Fig. 2. Factors that the epileptologists take into consideration when deciding if/when to taper AEDs in patients after epilepsy surgery. 
have not shown any factors to be consistently associated with an increased risk. In patients undergoing anterior temporal lobectomy, only the presence of interictal epileptiform discharges on postoperative EEG and absence of definite hippocampal sclerosis on pathology were predictive of unsuccessful AED withdrawal [14]. Age at surgery and duration of epilepsy are not considered by most epileptologists, although some studies suggest that they may be an important factor for determining relapse $[4,5]$.

In this survey of adult and pediatric epileptologists, the majority stated that they taper AEDs over 1-6 months. Other studies, including the surveys mentioned above, have not looked at this question. Thus, the ideal rate of AED tapering is unknown. A Cochrane review in 2006 only found one small study with weak methodology of 149 children that compared a rapid taper of 6 weeks to a long taper of 9 months $[15,16]$. Both groups had similar rates of seizure recurrence at various time points up to 5 years. These data suggest that the rate of AED tapering is unrelated to seizure recurrence, but further studies are needed to evaluate this.

When a breakthrough seizure does occur during tapering of AEDs, most of the respondents felt that seizure control could be reestablished, although $>20 \%$ felt this was not always the case. In a prospective study of patients undergoing temporal lobectomy, medications were withdrawn in 60/88 patients who had been seizure-free for at least a year [4]. Twenty had recurrence of seizures after withdrawal. Predictors of successful withdrawal of AEDs were younger age at surgery and shorter duration of epilepsy prior to surgery. Of note, in this study, 9/20 patients became seizure-free after restarting their medications; however, eleven did not, raising the concern that unsuccessful withdrawal of AEDs may lead to lack of seizure control even after AEDs are reinstituted.

The main limitation of our study is the relatively low response rate. It is possible that treatment preferences between responders and non-responders are different and that the responders may represent physicians with a special interest in AED withdrawal after epilepsy surgery. In addition, survey answers may not reflect true practice but instead an idealized version of it. A patient's desire to withdraw AEDs is frequently identified as an important consideration and likely influences this decision greatly. Another limitation is due to the fact that we purposefully limited the size of the survey in order to improve the likelihood of response. However, this necessitated questions that are somewhat general, which may miss some nuances that are important in such decisions. Despite the limitations, our survey highlights the practice variability regarding AED withdrawal after epilepsy surgery and the need for controlled studies to determine the ideal method for AED withdrawal. Because of the multiple variables involved in such a study, only a carefully designed, large, likely multicenter, study could answer such a question.

\section{References}

[1] McIntosh AM, Wilson SJ, Berkovic SF. Seizure outcome after temporal lobectomy: current research practice and findings. Epilepsia 2001;42(10):1288-307.

[2] Tellez-Zenteno JF, et al. Surgical outcomes in lesional and non-lesional epilepsy: a systematic review and meta-analysis. Epilepsy Res 2010;89(2-3):310-8.

[3] Tellez-Zenteno JF, et al. Long-term outcomes in epilepsy surgery: antiepileptic drugs, mortality, cognitive and psychosocial aspects. Brain 2007;130:334-45.

[4] Kim YD, et al. Antiepileptic drug withdrawal after successful surgery for intractable temporal lobe epilepsy. Epilepsia 2005;46(2):251-7.

[5] Park KI, et al. Withdrawal of antiepileptic drugs after neocortical epilepsy surgery. Ann Neurol 2010;67(2):230-8.

[6] Schiller Y, et al. Discontinuation of antiepileptic drugs after successful epilepsy surgery. Neurology 2000;54(2):346-9.

[7] Kerling F, et al. Drug withdrawal after successful epilepsy surgery: how safe is it? Epilepsy Behav 2009;15(4):476-80.

[8] Menon R, et al. Feasibility of antiepileptic drug withdrawal following extratemporal resective epilepsy surgery. Neurology 2012;79(8):770-6.

[9] Berg AT, et al. Stopping antiepileptic drugs after epilepsy surgery: a survey of US epilepsy center neurologists. Epilepsy Behav 2007;10(2):219-22.

[10] Tellez-Zenteno JF, et al. Discontinuation of antiepileptic drugs after successfu epilepsy surgery. A Canadian survey. Epilepsy Res 2012;102(1-2):23-33.

[11] Boshuisen $\mathrm{K}$, et al. Timing of antiepileptic drug withdrawal and long-term seizure outcome after paediatric epilepsy surgery (TimeToStop): a retrospective observational study. Lancet Neurol 2012;11(9):784-91.

[12] Murro AM, et al. Withdrawal of antiepileptic medications following epilepsy surgery. J Epilepsy 1991;4(1):39-42.

[13] Al-Kaylani M, et al. Seizure freedom off antiepileptic drugs after temporal lobe epilepsy surgery. Seizure-Eur J Epilepsy 2007;16(2):95-8.

[14] Rathore C, et al. How safe is it to withdraw antiepileptic drugs following successful surgery for mesial temporal lobe epilepsy? Epilepsia 2011;52(3):627-35.

[15] Ranganathan LN, Ramaratnam S. Rapid versus slow withdrawal of antiepileptic drugs. Cochrane Database Syst Rev 2006(2).

[16] Tennison M, et al. Discontinuing antiepileptic drugs in children with epilepsy - a comparison of a 6-week and a 9-month taper period. N Engl J Med 1994;330(20): 1407-10. 